October 29, 2018

hep-th/0607080

Imperial/TP/2006/JG/01

CERN-PH-TH/2006-129

HUTP-06/A0018

\title{
Obstructions to the Existence of Sasaki-Einstein Metrics
}

Jerome P. Gauntlett ${ }^{1,2}$, Dario Martelli ${ }^{3}$, James Sparks ${ }^{4,5}$ and Shing-Tung Yau ${ }^{4}$

1: Blackett Laboratory, Imperial College

London SW7 2AZ, U.K.

2: The Institute for Mathematical Sciences, Imperial College

London SW7 2PG, U.K.

3: Department of Physics, CERN Theory Unit

1211 Geneva 23, Switzerland

4: Department of Mathematics, Harvard University

One Oxford Street, Cambridge, MA 02138, U.S.A.

5: Jefferson Physical Laboratory, Harvard University

Cambridge, MA 02138, U.S.A.

\begin{abstract}
We describe two simple obstructions to the existence of Ricci-flat Kähler cone metrics on isolated Gorenstein singularities or, equivalently, to the existence of Sasaki-Einstein metrics on the links of these singularities. In particular, this also leads to new obstructions for Kähler-Einstein metrics on Fano orbifolds. We present several families of hypersurface singularities that are obstructed, including 3-fold and 4-fold singularities of ADE type that have been studied previously in the physics literature. We show that the AdS/CFT dual of one obstruction is that the $\mathrm{R}$-charge of a gauge invariant chiral primary operator violates the unitarity bound.
\end{abstract}




\section{Contents}

1 Introduction 1

2 The obstructions $\quad 5$

2.1 The Bishop obstruction . . . . . . . . . . . . . . . . . . . . . . . 6

2.2 The Lichnerowicz obstruction . . . . . . . . . . . . . . . . . 7

2.3 Smooth Fanos . . . . . . . . . . . . . . . . . . . . . . 9

$2.4 \mathrm{AdS} / \mathrm{CFT}$ interpretation . . . . . . . . . . . . . . . 11

3 Isolated hypersurface singularities $\quad 14$

3.1 The Bishop obstruction . . . . . . . . . . . . . . . . . . . . 16

3.2 The Lichnerowicz obstruction . . . . . . . . . . . . . . . . . . . . . . . 18

3.3 Sufficient conditions for existence . . . . . . . . . . . . . . . . . 18

4 A class of 3-fold examples 19

4.1 Obstructions . . . . . . . . . . . . . . . . . . . . . . . . 19

4.2 Cohomogeneity one metrics . . . . . . . . . . . . . . . . . . . . . . . . 20

4.3 Field theorv . . . . . . . . . . . . . . . . . . . . . . . 21

5 Other examples $\quad 23$

5.1 ADE 4-fold singularities . . . . . . . . . . . . . . . . . . . . . 24

5.2 Weighted actions on $\mathbb{C}^{n} \ldots \ldots \ldots \ldots \ldots$

6 Conclusions $\quad 26$

$\begin{array}{ll}\text { A Cohomogeneity one metrics } & 28\end{array}$

\section{Introduction}

The study of string theory and M-theory on singular manifolds is a very rich subject that has led to many important insights. For geometries that develop an isolated singularity, one can model the local behaviour using a non-compact manifold. In this case, a natural geometric boundary condition is for the metric to asymptote to a cone away from the singularity. This means that one studies a family of metrics that 
asymptotically approach the conical form

$$
g_{X}=\mathrm{d} r^{2}+r^{2} g_{L}
$$

with $\left(L, g_{L}\right)$ a compact Riemannian manifold. The dynamics of string theory or Mtheory on special holonomy manifolds that are developing an isolated conical singularity $X$, with metric (1.1), has proved to be an extremely intricate subject.

A particularly interesting setting is in the context of the AdS/CFT correspondence [1. The worldvolume theory of a large number of D3-branes placed at an isolated conical Calabi-Yau 3-fold singularity is expected to flow, at low energies, to a fourdimensional $\mathcal{N}=1$ superconformal field theory. In this case, the AdS/CFT conjecture states that this theory is dual to type IIB string theory on $\operatorname{AdS}_{5} \times L$ [2, 3, 4, 5. Similar remarks apply to M-theory on conical eight-dimensional singularities with special holonomy, which lead to superconformal theories in three dimensions that are dual to $\mathrm{AdS}_{4} \times L$, although far less is known about this situation.

The focus of this paper will be on conical Calabi-Yau singularities, by which we mean Ricci-flat Kähler metrics of the conical form (1.1). This gives, by definition, a Sasaki-Einstein metric on the base of the cone $L$. We tacitly assume that $L$ is simplyconnected which, although not entirely necessary, always ensures the existence of a globally defined Killing spinor on $L$. A central role is played by the Reeb vector field

$$
\xi=J\left(r \frac{\partial}{\partial r}\right)
$$

where $J$ denotes the complex structure tensor on the cone $X$. $\xi$ is holomorphic, Killing, and has constant norm on the link $L=\{r=1\}$ of the singularity at $r=0$. If the orbits of $\xi$ all close then $L$ has a $U(1)$ isometry, which necessarily acts locally freely, and the Sasakian structure is said to be either regular or quasi-regular if this action is free or not, respectively. The orbit space is in general a positively curved Kähler-Einstein orbifold $\left(V, g_{V}\right)$, which is a smooth manifold in the regular case. More generally, the generic orbits of $\xi$ need not close, in which case the Sasakian structure is said to be irregular. The AdS/CFT correspondence maps the symmetry generated by the Reeb vector field to the $\mathrm{R}$-symmetry of the dual CFT. Thus for the quasi-regular case the CFT has a $U(1)$ R-symmetry, whereas for the irregular case it has a non-compact $\mathbb{R}$ R-symmetry.

Given a Sasaki-Einstein manifold $\left(L, g_{L}\right)$, the cone $X$, as a complex variety, is an isolated Gorenstein singularity. If $X_{0}$ denotes $X$ with the singular point removed, 
we have $X_{0}=\mathbb{R}_{+} \times L$ with $r>0$ a coordinate on $\mathbb{R}_{+} \cdot X$ being Gorenstein means simply that there exists a nowhere zero holomorphic $(n, 0)$-form $\Omega$ on $X_{0}$. One may then turn things around and ask which isolated Gorenstein singularities admit SasakiEinstein metrics on their links. This is a question in algebraic geometry, and it is an extremely difficult one. To give some idea of how difficult this question is, let us focus on the quasi-regular case. Thus, suppose that $X$ has a holomorphic $\mathbb{C}^{*}$ action, with orbit space being a Fano ${ }^{1}$ manifold, or Fano orbifold, $V$. Then existence of a Ricci-flat Kähler cone metric on $X$, with conical symmetry generated by $\mathbb{R}_{+} \subset \mathbb{C}^{*}$, is well known to be equivalent to finding a Kähler-Einstein metric on $V$ - for a review, see [6]. Existence of Kähler-Einstein metrics on Fanos is a very subtle problem that is still unsolved. That is, a set of necessary and sufficient algebraic conditions on $V$ are not known in general. There are two well-known holomorphic obstructions, due to Matsushima [7] and Futaki [8. The latter was related to Sasakian geometry in 9] and is not in fact an obstruction from the Sasaki-Einstein point view. Specifically, it is possible to have a Fano $V$ that has non-zero Futaki invariant and thus does not admit a Kähler-Einstein metric, but nevertheless the link of the total space of the canonical bundle over $V$ can admit a Sasaki-Einstein metric - the point is simply that the Reeb vector field is not ${ }^{2}$ the one associated with the canonical bundle over $V$. It is also known that vanishing of these two obstructions is, in general, insufficient for there to exist a Kähler-Einstein metric on $V$. It has been conjectured in [1] that $V$ admits a Kähler-Einstein metric if and only if it is stable; proving this conjecture is currently a major research programme in geometry - see, for example, 12. Thus, one also expects the existence of Ricci-flat Kähler cone metrics on an isolated Gorenstein singularity $X$ to be a subtle problem. This issue has been overlooked in some of the physics literature, and it has sometimes been incorrectly assumed, or stated, that such conical Calabi-Yau metrics exist on particular singularities, as we shall discuss later.

The Reeb vector field contains a significant amount of information about the metric. For a fixed $X_{0}=\mathbb{R}_{+} \times L$, the Reeb vector field $\xi$ for a Sasaki-Einstein metric on $L$ satisfies a variational problem that depends only on the complex structure of $X$ [13, 9]. This is the geometric analogue of $a$-maximisation [14] in four dimensional

\footnotetext{
${ }^{1}$ We define a Fano orbifold $V$ to be a compact Kähler orbifold, such that the cohomology class of the Ricci-form in $H^{2}(V ; \mathbb{R})$ is represented by a positive $(1,1)$-form on $V$.

${ }^{2}$ This happens, for example, when $V=\mathbb{F}_{1}$ - the first del Pezzo surface. In this case both the Matsushima and Futaki theorems obstruct existence of a Kähler-Einstein metric on $V$, but there is nevertheless an irregular Sasaki-Einstein metric on the link in the total space of the canonical bundle over $V[10]$.
} 
superconformal field theories. This allows one, in principle and often in practice, to obtain $\xi$, and hence in particular the volume, of a Sasaki-Einstein metric on $L$ assuming that this metric exists.

Now, for any $(2 n-1)$-dimensional Einstein manifold $\left(L, g_{L}\right)$ with Ric $=2(n-1) g_{L}$, Bishop's theorem [15] (see also [16]) implies that the volume of $L$ is bounded from above by that of the round unit radius sphere. Thus we are immediately led to what we will call the Bishop obstruction to the existence of Sasaki-Einstein metrics:

If the volume of the putative Sasaki-Einstein manifold, calculated using the results of [13, 9], is greater than that of the round sphere, then the metric cannot exist.

It is not immediately obvious that this can ever happen, but we shall see later that this remarkably simple fact can often serve as a powerful obstruction. We will also discuss the AdS/CFT interpretation of this result.

The Reeb vector field $\xi$ also leads to a second possible obstruction. Given $\xi$ for a putative Sasaki-Einstein metric, it is a simple matter to show that holomorphic functions $f$ on the corresponding cone $X$ with definite charge $\lambda>0$,

$$
\mathcal{L}_{\xi} f=\lambda i f
$$

give rise to eigenfunctions of the Laplacian on the Sasaki-Einstein manifold with eigenvalue $\lambda(\lambda+2 n-2)$. Lichnerowicz's theorem [17] states that the smallest eigenvalue of this Laplacian is bounded from below by the dimension of the manifold, and this leads to the restriction $\lambda \geq 1$. Thus we have what we will call the Lichnerowicz obstruction:

If one can demonstrate the existence of a holomorphic function on $X$ with positive charge $\lambda<1$ with respect to the putative Reeb vector field $\xi$, one concludes that no Sasaki-Einstein metric can exist with this Reeb vector field.

Again, it is not immediately obvious that this can ever happen. Indeed, if $\xi$ is regular, so that the orbit space $V$ is a Fano manifold, we show that this cannot happen. Nevertheless, there are infinitely many examples of simple hypersurface singularities with non-regular Reeb vector fields that violate Lichnerowicz's bound.

We shall show that for Calabi-Yau 3-folds and 4-folds, the Lichnerowicz obstruction has a beautiful AdS/CFT interpretation: holomorphic functions on the cone $X$ are dual 
to chiral primary operators in the dual superconformal field theory. The Lichnerowicz bound then translates into the unitarity bound for the dimensions of the operators.

The plan of the rest of the paper is as follows. In section 2 we discuss the two obstructions in a little more detail. In section 3 we investigate the obstructions in the context of isolated quasi-homogeneous hypersurface singularities. We also compare our results with the sufficient conditions reviewed in [6] for existence of Sasaki-Einstein metrics on links of such singularities. In section 4 we show that some 3-fold examples discussed in [18] do not admit Ricci-flat Kähler cone metrics. We briefly discuss the implications for the dual field theory. The results of this section leave open the possibility ${ }^{3}$ of a single new cohomogeneity one Sasaki-Einstein metric on $S^{5}$ and we present some details of the relevant ODE that needs to be solved in an appendix. In section 5 we show that some of the 4-fold examples discussed in [20] also do not admit Ricci-flat Kähler cone metrics. Section 6 briefly concludes.

\section{The obstructions}

In this section we describe two obstructions to the existence of a putative SasakiEinstein metric on the link of an isolated Gorenstein singularity $X$ with Reeb vector field $\xi$. These are based on Bishop's theorem [15] and Lichnerowicz's theorem [17], respectively. We prove that the case when $\xi$ generates a freely acting circle action, with orbit space a Fano manifold $V$, is never obstructed by Lichnerowicz. We also give an interpretation of Lichnerowicz's bound in terms of the unitarity bound in field theory, via the AdS/CFT correspondence.

Let $X$ be an isolated Gorenstein singularity, and $X_{0}$ be the smooth part of $X$. We take $X_{0}$ to be diffeomorphic as a real manifold to $\mathbb{R}_{+} \times L$ where $L$ is compact, and let $r$ be a coordinate on $\mathbb{R}_{+}$with $r>0$, so that $r=0$ is the isolated singular point of $X$. We shall refer to $L$ as the link of the singularity. Since $X$ is Gorenstein, by definition there exists a nowhere zero holomorphic $(n, 0)$-form $\Omega$ on $X_{0}$.

Suppose that $X$ admits a Kähler metric that is a cone with respect to a homothetic vector field $r \partial / \partial r$, as in (1.1). This in particular means that $L$ is the orbit space of $r \partial / \partial r$ and $g_{L}$ is a Sasakian metric. The Reeb vector field is defined to be

$$
\xi=J\left(r \frac{\partial}{\partial r}\right)
$$

\footnotetext{
${ }^{3}$ Recently reference [19] appeared. The conclusions of the latter imply that this solution does not in fact exist.
} 
In the special case that the Kähler metric on $X$ is Ricci-flat, the case of central interest, $\left(L, g_{L}\right)$ is Sasaki-Einstein and we have

$$
\mathcal{L}_{\xi} \Omega=n i \Omega
$$

since $\Omega$ is homogeneous of degree $n$ under $r \partial / \partial r$. This fixes the normalisation of $\xi$.

\subsection{The Bishop obstruction}

The volume $\operatorname{vol}\left(L, g_{L}\right)$ of a Sasakian metric on the link $L$ depends only on the Reeb vector field [9]. Thus, specifying a Reeb vector field $\xi$ for a putative Sasaki-Einstein metric on $L$ is sufficient to specify the volume, assuming that the metric in fact exists. We define the normalised volume as

$$
V(\xi)=\frac{\operatorname{vol}\left(L, g_{L}\right)}{\operatorname{vol}\left(S^{2 n-1}\right)}
$$

where $\operatorname{vol}\left(S^{2 n-1}\right)$ is the volume of the round sphere. Since Bishop's theorem [15] (see also [16]) implies that for any $(2 n-1)$-dimensional Einstein manifold $\left(L, g_{L}\right)$ with $\operatorname{Ric}=2(n-1) g_{L}$

$$
\operatorname{vol}\left(L, g_{L}\right) \leq \operatorname{vol}\left(S^{2 n-1}\right)
$$

we immediatley have

Bishop obstruction: Let $(X, \Omega)$ be an isolated Gorenstein singularity with link $L$ and putative Reeb vector field $\xi$. If $V(\xi)>1$ then $X$ admits no Ricci-flat Kähler cone metric with Reeb vector field $\xi$. In particular $L$ does not admit a Sasaki-Einstein metric with this Reeb vector field.

There are a number of methods for computing the normalised volume $V(\xi)$. For quasiregular $\xi$, the volume $V(\xi)$ is essentially just a Chern number, which makes it clear that $V(\xi)$ is a holomorphic invariant. In general, one can compute $V(\xi)$ as a function of $\xi$, and a number of different formulae have been derived in [13, 9]. In [9] a general formula for the normalised volume $V(\xi)$ was given that involves (partially) resolving the singularity $X$ and applying localisation. For toric Sasakian manifolds there is a simpler formula [13], giving the volume in terms of the toric data defining the singularity. In this paper we shall instead exploit the fact that the volume $V(\xi)$ can be extracted from a limit of a certain index-character [9]; this is easily computed algebraically for isolated hypersurface singularities, which shall constitute our main set of examples in this paper. 
We briefly recall some of the details from [9]. Suppose we have a holomorphic $\left(\mathbb{C}^{*}\right)^{r}$ action on $X$. We may define the character

$$
C(\mathbf{q}, X)=\operatorname{Tr} \mathbf{q}
$$

as the trace ${ }^{4}$ of the action of $\mathbf{q} \in\left(\mathbb{C}^{*}\right)^{r}$ on the holomorphic functions on $X$. Holomorphic functions $f$ on $X$ that are eigenvectors of the induced $\left(\mathbb{C}^{*}\right)^{r}$ action

$$
\left(\mathbb{C}^{*}\right)^{r}: f \rightarrow \mathbf{q}^{\mathbf{m}} f
$$

with eigenvalue $\mathbf{q}^{\mathbf{m}}=\prod_{a=1}^{r} q_{a}^{m_{a}}$ form a vector space over $\mathbb{C}$ of dimension $n_{\mathbf{m}}$. Each eigenvalue then contributes $n_{\mathbf{m}} \mathbf{q}^{\mathbf{m}}$ to the trace (2.5). Let $\zeta_{a}$ form a basis for the Lie algebra of $U(1)^{r} \subset\left(\mathbb{C}^{*}\right)^{r}$, and write the Reeb vector field as

$$
\xi=\sum_{a=1}^{r} b_{a} \zeta_{a} .
$$

Then the volume of a Sasakian metric on $L$ with Reeb vector field $\xi$, relative to that of the round sphere, is given by

$$
V(\xi)=\lim _{t \rightarrow 0} t^{n} C\left(q_{a}=\exp \left(-t b_{a}\right), X\right)
$$

In general, the right hand side of this formula may be computed by partially resolving $X$ and using localisation. However, for isolated quasi-homogeneous hypersurface singularities it is straightforward to compute this algebraically.

In addition, it was shown in [9] that the Reeb vector field for a Sasaki-Einstein metric on $L$ extremises $V$ as a function of the $b_{a}$, subject to the constraint (2.2). This is a geometric analogue of $a$-maximisation [14] in superconformal field theories.

\subsection{The Lichnerowicz obstruction}

Let $f$ be a holomorphic function on $X$ with

$$
\mathcal{L}_{\xi} f=\lambda i f
$$

where $\mathbb{R} \ni \lambda>0$, and we refer to $\lambda$ as the charge of $f$ under $\xi$. Since $f$ is holomorphic, this immediately implies that

$$
f=r^{\lambda} \tilde{f}
$$

\footnotetext{
${ }^{4}$ As in [9], we don't worry about where this trace converges, since we are mainly interested in the behaviour near a certain pole.
} 
where $\tilde{f}$ is homogeneous degree zero under $r \partial / \partial r$ - that is, $\tilde{f}$ is the pull-back to $X$ of a function on the link $L$. Moreover, since $\left(X, g_{X}\right)$ is Kähler,

$$
\nabla_{X}^{2} f=0
$$

where $-\nabla_{X}^{2}$ is the Laplacian on $\left(X, g_{X}\right)$. For a metric cone, this is related to the Laplacian on the link $\left(L, g_{L}\right)$ at $r=1$ by

$$
\nabla_{X}^{2}=\frac{1}{r^{2}} \nabla_{L}^{2}+\frac{1}{r^{2 n-1}} \frac{\partial}{\partial r}\left(r^{2 n-1} \frac{\partial}{\partial r}\right)
$$

From this, one sees that

$$
-\nabla_{L}^{2} \tilde{f}=E \tilde{f}
$$

where

$$
E=\lambda[\lambda+(2 n-2)] .
$$

Thus any holomorphic function $f$ of definite charge under $\xi$, or equivalently degree under $r \partial / \partial r$, corresponds to an eigenfunction of the Laplacian on the link. The charge $\lambda$ is then related simply to the eigenvalue $E$ by the above formula (2.14).

By assumption, $\left(X, g_{X}\right)$ is Ricci-flat Kähler, which implies that $\left(L, g_{L}\right)$ is Einstein with Ricci curvature $2 n-2$. The first non-zero eigenvalue $E_{1}>0$ of $-\nabla_{L}^{2}$ is bounded from below:

$$
E_{1} \geq 2 n-1
$$

This is Lichnerowicz's theorem [17. Moreover, equality holds if and only if $\left(L, g_{L}\right)$ is isometric to the round sphere $S^{2 n-1}$ [21]. This is important as we shall find examples of links, that are not even diffeomorphic to the sphere, which hit this bound. From (2.14), we immediately see that Lichnerowicz's bound becomes $\lambda \geq 1$.

This leads to a potential holomorphic obstruction to the existence of Sasaki-Einstein metrics:

Lichnerowicz obstruction: Let $(X, \Omega)$ be an isolated Gorenstein singularity with link $L$ and putative Reeb vector field $\xi$. Suppose that there exists a holomorphic function $f$ on $X$ of positive charge $\lambda<1$ under $\xi$. Then $X$ admits no Ricci-flat Kähler cone metric with Reeb vector field $\xi$. In particular $L$ does not admit a Sasaki-Einstein metric with this Reeb vector field. 
As we stated earlier, it is not immediately clear that this can ever happen. In fact, there are examples of hypersurface singularities where this serves as the only obvious simple obstruction, as we explain later. However, in the next subsection we treat a situation where Lichnerowicz never obstructs.

Before concluding this subsection we note that the volume of a Sasakian metric on $L$ with Reeb vector field $\xi$ is also related to holomorphic functions on $X$ of definite charge, as we briefly reviewed in the previous subsection. In fact we may write (2.8) as

$$
V(\xi)=\lim _{t \rightarrow 0} t^{n} \operatorname{Tr} \exp \left(-t \mathcal{L}_{r \partial / \partial r}\right)
$$

where $r \partial / \partial r=-J(\xi)$. Here the trace denotes a trace of the action of $\mathcal{L}_{r \partial / \partial r}$ on the holomorphic functions on $X$. Thus a holomorphic function $f$ of charge $\lambda$ under $\xi$ contributes $\exp (-t \lambda)$ to the trace. That (2.16) agrees with (2.8) follows from the fact that we can write $\lambda=(\mathbf{b}, \mathbf{m})$. Given our earlier discussion relating $\lambda$ to eigenvalues of the Laplacian on $L$, the above trace very much resembles the trace of the heat kernel, also known as the partition function, on $L$. In fact, since it is a sum over only holomorphic eigenvalues, we propose to call it the holomorphic partition function. The fact that the volume of a Riemannian manifold appears as a pole in the heat kernel is well known 22, and (2.16) can be considered a holomorphic Sasakian analogue.

Notice then that the Lichnerowicz obstruction involves holomorphic functions on $X$ of small charge with respect to $\xi$, whereas the Bishop obstruction is a statement about the volume, which is determined by the asymptotic growth of holomorphic functions on $X$.

\subsection{Smooth Fanos}

Let $V$ be a smooth Fano Kähler manifold. Let $K$ denote the canonical line bundle over $V$. By definition, $K^{-1}$ is an ample holomorphic line bundle, which thus specifies a positive class

$$
c_{1}\left(K^{-1}\right)=-c_{1}(K) \in H^{2}(V ; \mathbb{Z}) \cap H^{1,1}(V ; \mathbb{R}) \cong \operatorname{Pic}(V)
$$

Recall here that $\operatorname{Pic}(V)$ is the group of holomorphic line bundles on $V$. Let $I(V)$ denote the largest positive integer such that $c_{1}\left(K^{-1}\right) / I(V)$ is an integral class in $\operatorname{Pic}(V) . I(V)$ is called the Fano index of $V$. For example, $I\left(\mathbb{C P}^{2}\right)=3, I\left(\mathbb{C P}^{1} \times \mathbb{C P}^{1}\right)=2, I\left(\mathbb{F}_{1}\right)=1$. Let $\mathcal{L}$ be the holomorphic line bundle $\mathcal{L}=K^{1 / I(V)}$, which is primitive in $\operatorname{Pic}(V)$ by construction. Denote the total space of the unit circle bundle in $\mathcal{L}$ by $L$ - this is our 
link. We thus have a circle bundle

$$
S^{1} \hookrightarrow L \rightarrow V
$$

where $\mathcal{L}$ is the associated line bundle. If $V$ is simply-connected then $L$ is also simplyconnected, as follows from the Gysin sequence of the fibration (2.18). Note that $V$ admits a Kähler-Einstein metric if and only if $L$ admits a regular Sasaki-Einstein metric with Reeb vector field that rotates the $S^{1}$ fibre of (2.18).

$X$ is obtained from the total space of $\mathcal{L}$ by collapsing (or deleting, to obtain $X_{0}$ ) the zero section. Holomorphic functions on $X$ of definite charge are then in 1-1 correspondence with global sections of $\mathcal{L}^{-k}$, which are elements of the group $H^{0}\left(\mathcal{O}\left(\mathcal{L}^{-k}\right)\right)$. Let $\zeta$ be the holomorphic vector field on $X$ that rotates the fibre of $\mathcal{L}$ with weight one. That is, if $s \in H^{0}\left(\mathcal{O}\left(\mathcal{L}^{-1}\right)\right)$ is a holomorphic section of the ample line bundle $\mathcal{L}^{-1}$, viewed as a holomorphic function on $X$, then

$$
\mathcal{L}_{\zeta} s=i s .
$$

Since $K=\mathcal{L}^{I(V)}$ is the canonical bundle of $V$, it follows that the correctly normalised Reeb vector field is (see, for example, [9])

$$
\xi=\frac{n}{I(V)} \zeta .
$$

We briefly recall why this is true. Let $\psi$ be a local coordinate such that $\xi=\partial / \partial \psi$. Then $n \psi / I(V)$ is a local coordinate on the circle fibre of (2.18) with period $2 \pi$. This follows since locally the contact one-form of the Sasakian manifold is $\eta=\mathrm{d} \psi-A$, where $A / n$ is a connection on the canonical bundle of $V$.

Holomorphic functions of smallest positive charge obviously correspond to $k=1$. Any section $s \in H^{0}\left(\mathcal{O}\left(\mathcal{L}^{-1}\right)\right)$ then has charge

$$
\lambda=\frac{n}{I(V)}
$$

under $\xi$. However, it is well known (see, for example, [23, page 245) that for smooth Fanos $V$ we have $I(V) \leq n$, with $I(V)=n$ if and only if $V=\mathbb{C P}^{n-1}$. Thus, in this situation, we always have $\lambda \geq 1$ and Lichnerowicz never obstructs.

\section{Lichnerowicz's theorem can only obstruct for non-regular Reeb vector fields.}

We expect a similar statement to be true for the Bishop bound. For a regular SasakiEinstein manifold with Reeb vector field $\xi$ and orbit space a Fano manifold $V$, Bishop's 
bound may be written

$$
I(V) \int_{V} c_{1}(V)^{n-1} \leq n \int_{\mathbb{C P}^{n-1}} c_{1}\left(\mathbb{C P}^{n-1}\right)^{n-1}=n^{n} .
$$

It seems reasonable to expect the topological statement (2.22) to be true for any Fano manifold $V$, so that Bishop never obstructs in the regular case, although we are unaware of any proof. Interestingly, this is closely related to a standard conjecture in algebraic

geometry, that bounds $\int_{V} c_{1}(V)^{n-1}$ from above by $n^{n-1}$ for any Fano manifold $V$, with equality if and only if $V=\mathbb{C P}^{n-1}$. In general, this stronger statement is false (see [23], page 251), although it is believed to be true in the special case that $V$ has Picard number one, i.e. $\operatorname{rank}(\operatorname{Pic}(V))=1$. This has recently been proven up to dimension $n=5$ [24]. It would be interesting to investigate (2.22) further.

\subsection{AdS/CFT interpretation}

In this section we show that the Lichnerowicz obstruction has a very natural interpretation in the AdS/CFT dual field theory, in terms of a unitarity bound. We also briefly discuss the Bishop bound.

Recall that every superconformal field theory possesses a supergroup of symmetries and that the AdS/CFT duality maps this to the superisometries of the dual geometry. In particular, in the context of Sasaki-Einstein geometry, it maps the $\mathrm{R}$-symmetry in the field theory to the isometry generated by the Reeb vector field $\xi$, and the $\mathrm{R}$-charges of operators in the field theory are proportional to the weights under $\xi$. Generically, Kaluza-Klein excitations in the geometry correspond to gauge invariant operators in the field theory. These operators are characterised by their scaling dimensions $\Delta$. The supersymmetry algebra then implies that a general operator satisfies a BPS bound relating the dimension to the $\mathrm{R}$-charge $R: \Delta \geq(d-1) R / 2$. When this bound is saturated the corresponding BPS operators belong to short representations of the supersymmetry algebra, and in particular are chiral. Here we will only consider scalar gauge invariant operators which are chiral.

It is well known that for any conformal field theory, in arbitrary dimension $d$, the scaling dimensions of all operators are bounded as a consequence of unitarity. In particular, for scalar operators, we have

$$
\Delta \geq \frac{d-2}{2}
$$

In section 2.2 we have argued that a necessary condition for the existence of a SasakiEinstein metric is that the charge $\lambda>0$ of any holomorphic function on the corre- 
sponding Calabi-Yau cone must satisfy the bound

$$
\lambda \geq 1
$$

In the following, we will show that these two bounds coincide.

We start with a gauge theory realised on the world-volume of a large number of D3 branes, placed at a 3 -fold Gorenstein singularity $X$. The affine variety $X$ can then be thought of as (part of) the moduli space of vacua of this gauge theory. In particular, the holomorphic functions, defining the coordinate ring of $X$, correspond to (scalar) elements of the chiral ring of the gauge theory [25]. Recalling that an $\operatorname{AdS}_{4 / 5} \times L^{7 / 5}$ solution arises as the near-horizon limit of a large number of branes at a Calabi-Yau 4-fold/3-fold conical singularity, it is clear that the weights $\lambda$ of these holomorphic functions under the action of $r \partial / \partial r$ must be proportional to the scaling dimensions $\Delta$ of the dual operators, corresponding to excitations in AdS space. We now make this relation more precise.

According to the AdS/CFT dictionary [26, 27, a generic scalar excitation $\Phi$ in AdS obeying

$$
\left(\square_{\mathrm{AdS}_{d+1}}-m^{2}\right) \Phi=0
$$

and which behaves like $\rho^{-\Delta}$ near the boundary of $\operatorname{AdS}(\rho \rightarrow \infty)$, is dual to an operator in the dual CFT with scaling dimension

$$
m^{2}=\Delta(\Delta-d) \Rightarrow \Delta_{ \pm}=\frac{d}{2} \pm \sqrt{\frac{d^{2}}{4}+m^{2}} .
$$

More precisely, for $m^{2} \geq-d^{2} / 4+1$ the dimension of the operator is given by $\Delta_{+}$. However, for $-d^{2} / 4<m^{2}<-d^{2} / 4+1$ one can take either $\Delta_{ \pm}$and these will correspond to inequivalent CFTs [28, 29]. Notice that $\Delta_{+}$is always well above the bound implied by unitarity. On the other hand, $\Delta_{-}$saturates this bound for $m^{2}=-d^{2} / 4+1$.

The values for $m^{2}$ can be obtained from the eigenvalues $E$ of the scalar Laplacian $-\nabla_{L}^{2}$ on the internal manifold $L$ by performing a Kaluza-Klein analysis. The modes corresponding to the chiral primary operators have been identified in the literature in the context of a more general analysis for Einstein manifolds; see [28, 30] for type IIB supergravity compactified on $L^{5}$, and [31, 32] for M-theory compactified on $L^{7}$.

Consider first $d=4$ (i.e. $n=3$ ). The supergravity modes dual to chiral operators are a mixture of the trace mode of the internal metric and the RR four-form and lead to 33, 28, 30,

$$
m^{2}=E+16-8 \sqrt{E+4} .
$$


Combining this with (2.14) it follows that

$$
\Delta_{ \pm}=2 \pm|\lambda-2|
$$

so that $\Delta=\lambda$, providing that we take $\Delta_{-}$for $\lambda<2$ and $\Delta_{+}$for $\lambda \geq 2$. Notice that for $\lambda=2, \Delta_{+}=\Delta_{-}$, and this corresponds to the Breitenlohner-Freedman bound $m^{2}(\lambda=2)=-4$ for stability in $\mathrm{AdS}_{5}$.

The case $d=3$ (i.e. $n=4$ ), relevant for $\mathrm{AdS}_{4} \times L^{7}$ geometries, is similar. The scalar supergravity modes corresponding to chiral primaries [31, 32, are again a mixture of the metric trace and the three-form potential [34], and fall into short $\mathcal{N}=2$ multiplets. Their masses are given $b^{5}$ [34, 35, 32,

$$
m^{2}=\frac{E}{4}+9-3 \sqrt{E+9} .
$$

Combing this with (2.14) it follows that

$$
\Delta_{ \pm}=\frac{1}{2}(3 \pm|\lambda-3|)
$$

so that $\Delta=\frac{1}{2} \lambda$, providing that we take $\Delta_{-}$for $\lambda<3$ and $\Delta_{+}$for $\lambda \geq 3$. Once again the switching of the two branches occurs at the Breitenlohner-Freedman bound $m^{2}(\lambda=3)=-9 / 4$ for stability in $\mathrm{AdS}_{4}$.

In summary, we have shown that

$$
\Delta=\left\{\begin{array}{cc}
\lambda & \text { for } d=4 \\
\frac{1}{2} \lambda & \text { for } d=3
\end{array} .\right.
$$

Thus in both cases relevant for AdS/CFT the Lichnerowicz bound $\lambda \geq 1$ is equivalent to the unitarity bound (2.23).

The Bishop bound also has a direct interpretation in field theory. Recall that the volume of the Einstein 5 -manifold $\left(L, g_{L}\right)$ is related to the exact a central charge of the dual four dimensional conformal field theory via [36] (see also [37])

$$
a(L)=\frac{\pi^{3} N^{2}}{4 \operatorname{vol}\left(L, g_{L}\right)}
$$

where $N$ is the number of D3-branes. The Bishop bound then implies that

$$
a(L) \geq \frac{N^{2}}{4}=a(\mathcal{N}=4)
$$

\footnotetext{
${ }^{5}$ Note that the mass formulae in [35] are relative to the operator $\square_{A d S_{4}}-32$. Moreover, the factor of four mismatch between their $m^{2}$ and ours is simply due to the fact that it is actually $m^{2} R^{2}$ that enters in (2.26), and the radius of $\mathrm{AdS}_{4}$ is $1 / 2$ that of $\mathrm{AdS}_{5}$.
} 
where $N^{2} / 4$ is the central charge of $\mathcal{N}=4$ super Yang-Mills theory. One can give a heuristic argument for this inequality, as follows ${ }^{6}$. By appropriately Higgsing the dual field theory, and then integrating out the massive fields, one expects to be able to flow to $\mathcal{N}=4$ super Yang-Mills theory. This is because the Higgsing corresponds to moving the D3-branes away from the singular point to a smooth point of the cone, at which the near horizon geometry becomes $A d S_{5} \times S^{5}$. Since the number of massless degrees of freedom is expected to decrease in such a process, we also expect that the $a$ central charge to decrease. This would then explain the inequality (2.33).

\section{Isolated hypersurface singularities}

In this section we describe links of isolated quasi-homogeneous hypersurface singularities. These provide many simple examples of both obstructions.

Let $w_{i} \in \mathbb{Z}_{+}, i=1, \ldots, n+1$, be a set of positive weights. We denote these by a vector $\mathbf{w} \in\left(\mathbb{Z}_{+}\right)^{n+1}$. This defines an action of $\mathbb{C}^{*}$ on $\mathbb{C}^{n+1}$ via

$$
\left(z_{1}, \ldots, z_{n+1}\right) \mapsto\left(q^{w_{1}} z_{1}, \ldots, q^{w_{n+1}} z_{n+1}\right)
$$

where $q \in \mathbb{C}^{*}$. Without loss of generality one can take the set $\left\{w_{i}\right\}$ to have no common factor. This ensures that the above $\mathbb{C}^{*}$ action is effective. However, for the most part, this is unnecessary for our purposes and we shall not always do this. Let

$$
F: \mathbb{C}^{n+1} \rightarrow \mathbb{C}
$$

be a quasi-homogeneous polynomial on $\mathbb{C}^{n+1}$ with respect to $\mathbf{w}$. This means that $F$ has definite degree $d$ under the above $\mathbb{C}^{*}$ action:

$$
F\left(q^{w_{1}} z_{1}, \ldots, q^{w_{n+1}} z_{n+1}\right)=q^{d} F\left(z_{1}, \ldots, z_{n+1}\right)
$$

Moreover we assume that the affine algebraic variety

$$
X=\{F=0\} \subset \mathbb{C}^{n+1}
$$

is smooth everywhere except at the origin $(0,0, \ldots, 0)$. For obvious reasons, such $X$ are called isolated quasi-homogeneous hypersurface singularities. The corresponding link $L$ is the intersection of $X$ with the unit sphere in $\mathbb{C}^{n+1}$ :

$$
\sum_{i=1}^{n+1}\left|z_{i}\right|^{2}=1 .
$$

\footnotetext{
${ }^{6}$ We thank Ken Intriligator for this argument.
} 
A particularly nice set of such singularities are provided by so-called Brieskorn-Pham singularities. These take the particular form

$$
F=\sum_{i=1}^{n+1} z_{i}^{a_{i}}
$$

with $\mathbf{a} \in\left(\mathbb{Z}_{+}\right)^{n+1}$. Thus the weights of the $\mathbb{C}^{*}$ action are given by $w_{i}=d / a_{i}$. The corresponding hypersurface singularities $X$ are always isolated, as is easily checked. Moreover, the topology of the links $L$ are also extremely well understood - see [39] for a complete description of the homology groups of $L$. In particular, $L$ is known to be $(n-2)$-connected, meaning that the homotopy groups are $\pi_{a}(L)=0$ for all $a=1, \ldots, n-2$.

Returning to the general case, we may define a nowhere zero holomorphic $(n, 0)$-form $\Omega$ on the smooth part of $X$ by

$$
\Omega=\frac{\mathrm{d} z_{1} \wedge \cdots \wedge \mathrm{d} z_{n}}{\partial F / \partial z_{n+1}}
$$

This defines $\Omega$ on the patch where $\partial F / \partial z_{n+1} \neq 0$. One has similar expressions on patches where $\partial F / \partial z_{i} \neq 0$ for each $i$, and it is simple to check that these glue together into a nowhere zero form $\Omega$. Thus all such $X$ are Gorenstein, and moreover they come equipped with a holomorphic $\mathbb{C}^{*}$ action by construction. The orbit space of this $\mathbb{C}^{*}$ action, or equivalently the orbit space of $U(1) \subset \mathbb{C}^{*}$ on the link, is a complex orbifold $V$. In fact, $V$ is the weighted variety defined by $\{F=0\}$ in the weighted projective space $\mathbb{W} \mathbb{C P}_{\left[w_{1}, w_{2}, \ldots, w_{n+1}\right]}^{n}$. The latter is the quotient of the non-zero vectors in $\mathbb{C}^{n+1}$ by the weighted $\mathbb{C}^{*}$ action

$$
\mathbb{W} \mathbb{C P}_{\left[w_{1}, w_{2}, \ldots, w_{n+1}\right]}^{n}=\left(\mathbb{C}^{n+1} \backslash\{(0,0, \ldots, 0)\}\right) / \mathbb{C}^{*}
$$

and is a complex orbifold with a natural Kähler orbifold metric, up to scale, induced from Kähler reduction of the flat metric on $\mathbb{C}^{n+1}$. It is not difficult to show that $V$ is a Fano orbifold if and only if

$$
|\mathbf{w}|-d>0
$$

where $|\mathbf{w}|=\sum_{i=1}^{n+1} w_{i}$. To see this, first notice that $|\mathbf{w}|-d$ is the charge of $\Omega$ under $U(1) \subset \mathbb{C}^{*}$. To be precise, if $\zeta$ denotes the holomorphic vector field on $X$ with

$$
\mathcal{L}_{\zeta} z_{j}=w_{j} i z_{j}
$$

for each $j=1, \ldots, n+1$, then

$$
\mathcal{L}_{\zeta} \Omega=(|\mathbf{w}|-d) i \Omega
$$


Positivity of this charge $|\mathbf{w}|-d$ then implies [9] that the cohomology class of the natural Ricci-form induced on $V$ is represented by a positive $(1,1)$-form, which is the definition that $V$ is Fano.

If there exists a Ricci-flat Kähler metric on $X$ which is a cone under $\mathbb{R}_{+} \subset \mathbb{C}^{*}$, then the correctly normalised Reeb vector field is thus

$$
\xi=\frac{n}{|\mathbf{w}|-d} \zeta
$$

We emphasise that here we will focus on the possible (non-)existence of a SasakiEinstein metric on the link $L$ which has this canonical vector field as its Reeb vector field. It is possible that such metrics are obstructed, but that there exists a SasakiEinstein metric on $L$ with a different Reeb vector field. This may be investigated using the results of [9]. In particular we shall come back to this point for a class of 3-fold examples in section 4.

\subsection{The Bishop obstruction}

A general formula for the volume of a Sasaki-Einstein metric on the link of an isolated quasi-homogeneous hypersurface singularity was given in [40]. Strictly speaking, this formula was proven only when the Fano $V$ is well-formed. This means that the orbifold loci of $V$ are at least complex codimension two. When $V$ is not well-formed, the singular sets of $V$ considered as an orbifold and as an algebraic variety are in fact different. A simple example is the weighted projective space $\mathbb{W} \mathbb{C P}_{[p, q]}^{1}$ where $\operatorname{hcf}(p, q)=$ 1. As an orbifold, this is topologically a $2-$ sphere with conical singularities at the north and south poles of polar angle $2 \pi / p$ and $2 \pi / q$, respectively. As an algebraic variety, this weighted projective space is just $\mathbb{C P}^{1}$ since $\mathbb{C} / \mathbb{Z}_{p}=\mathbb{C}$. In fact, as a manifold it is diffeomorphic to $S^{2}$, for the same reason. When we say Kähler-Einstein orbifold metric, we must keep track of this complex codimension one orbifold data in the nonwell-formed case. For further details, the reader is directed to the review [6].

Assuming that there exists a Sasaki-Einstein metric with Reeb action $U(1) \subset \mathbb{C}^{*}$, then the volume of this link when $V$ is well-formed is given by [40]

$$
\operatorname{vol}(L)=\frac{2 d}{w(n-1) !}\left(\frac{\pi(|\mathbf{w}|-d)}{n}\right)^{n} .
$$

Here $w=\prod_{i=1}^{n+1} w_{i}$ denotes the product of the weights.

Using the earlier formula (2.8), we may now give an alternative derivation of this formula. The advantage of this approach is that, in contrast to [40, we never descend 
to the orbifold $V$. This allows us to dispense with the well-formed condition, and show that (3.13) holds in general. The authors of [40] noted that their formula seemed to apply to the general case.

Let us apply (2.8) to isolated quasi-homogeneous hypersurface singularities. Let $q \in \mathbb{C}^{*}$ denote the weighted action on $X$. We may compute the character $C(q, X)$ rather easily, since holomorphic functions on $X$ descend from holomorphic functions on $\mathbb{C}^{n+1}$, and the trace over the latter is simple to compute. A discussion of precisely this problem may be found in [41]. According to the latter reference, the character is simply

$$
C(q, X)=\frac{1-q^{d}}{\prod_{i=1}^{n+1}\left(1-q^{w_{i}}\right)} .
$$

The limit (2.8) is straightforward to take, giving the normalised volume

$$
V(\xi)=\frac{d}{w b^{n}}
$$

where, as above,

$$
\xi=b \zeta
$$

and $\zeta$ generates the $U(1) \subset \mathbb{C}^{*}$ action. Thus, from our earlier discussion on the charge of $\Omega$, we have

$$
b=\frac{n}{|\mathbf{w}|-d}
$$

giving

$$
\operatorname{vol}(L)=\frac{d(|\mathbf{w}|-d)^{n}}{w n^{n}} \operatorname{vol}\left(S^{2 n-1}\right)
$$

Restoring

$$
\operatorname{vol}\left(S^{2 n-1}\right)=\frac{2 \pi^{n}}{(n-1) !}
$$

we thus obtain the result (3.13).

Bishop's theorem then requires, for existence of a Sasaki-Einstein metric on $L$ with Reeb vector field $\xi$ generating the canonical $U(1)$ action,

$$
d(|\mathbf{w}|-d)^{n} \leq w n^{n}
$$

We shall see that infinitely many isolated quasi-homogeneous hypersurface singularities with Fano $V$ violate this inequality. 


\subsection{The Lichnerowicz obstruction}

As we already mentioned, holomorphic functions on $X$ are simply restrictions of holomorphic functions on $\mathbb{C}^{n+1}$. Thus the smallest positive charge holomorphic function is $z_{m}$, where $m \in\{1, \ldots, n+1\}$ is such that

$$
w_{m}=\min \left\{w_{i}, i=1, \ldots, n+1\right\} .
$$

Of course, $m$ might not be unique, but this is irrelevant since all such $z_{m}$ have the same charge in any case. This charge is

$$
\lambda=\frac{n w_{m}}{|\mathbf{w}|-d}
$$

and thus the Lichnerowicz obstruction becomes

$$
|\mathbf{w}|-d \leq n w_{m}
$$

Moreover, this bound can be saturated if and only if $X$ is $\mathbb{C}^{n}$ with its flat metric.

It is again clearly trivial to construct many examples of isolated hypersurface singularities that violate this bound.

\subsection{Sufficient conditions for existence}

In a series of works by Boyer, Galicki and collaborators, many examples of SasakiEinstein metrics have been shown to exist on links of isolated quasi-homogeneous hypersurface singularities of the form (3.6). Weighted homogeneous perturbations of these singularities can lead to continuous families of Sasaki-Einstein metrics. For a recent review of this work, we refer the reader to [6] and references therein.

Existence of these metrics is proven using the continuity method. One of the sufficient (but far from necessary) conditions for there to exist a Sasaki-Einstein metric is that the weights satisfy the condition [6]

$$
|\mathbf{w}|-d<\frac{n}{(n-1)} w_{m} .
$$

In particular, for $n>2$ this implies that

$$
|\mathbf{w}|-d<n w_{m}
$$

which is precisely Lichnerowicz's bound. Curiously, for $n=2$ the Lichnerowicz bound and (3.24) are the same, although this case is rather trivial. 


\section{A class of 3 -fold examples}

Our first set of examples are given by the 3 -fold singularities with weights $\mathbf{w}=$ $(k, k, k, 2)$ and polynomial

$$
F=\sum_{i=1}^{3} z_{i}^{2}+z_{4}^{k}
$$

where $k$ is a positive integer. The corresponding isolated hypersurface singularities $X_{k}=\{F=0\}$ are of Brieskorn-Pham type. Notice that $X_{1}=\mathbb{C}^{3}$ and $X_{2}$ is the ordinary double point singularity, better known to physicists as the conifold. Clearly, both of these admit Ricci-flat Kähler cone metrics and moreover, the Sasaki-Einstein metrics are homogeneous.

The differential topology of the links $L_{k}$ can be deduced using the results of [39], together with Smale's theorem for 5-manifolds. In particular, for $k$ odd, the link $L_{k}$ is diffeomorphic to $S^{5}$. For $k=2 p$ even, one can show that $L_{2 p} \cong S^{2} \times S^{3}$ (alternatively, see Lemma 7.1 of [42]).

The Fanos $V_{k}$ are not well-formed for $k>2$. In fact, the subvariety $z_{4}=0$ in $V_{k}$ is a copy of $\mathbb{C P}^{1}$, which is a locus of $\mathbb{Z}_{k}$ orbifold singularities for $k$ odd, and $\mathbb{Z}_{k / 2}$ orbifold singularities for $k$ even. As algebraic varieties, all the odd $k$ are equivalent to $\mathbb{C P}^{2}$, and all the even $k$ are equivalent to $\mathbb{C P}^{1} \times \mathbb{C P}^{1}$. As orbifolds, they are clearly all distinct.

\subsection{Obstructions}

These singularities have appeared in the physics literature [18] where it was assumed that all $X_{k}$ admitted conical Ricci-flat Kähler metrics, with Reeb action corresponding to the canonical $U(1)$ action. In fact, it is trivial to show that the Bishop bound (3.20) is violated for all $k>20$. Moreover, the Lichnerowicz bound (3.23) is even sharper: for $k \geq 2, z_{4}$ has smallest ${ }^{7}$ charge under $\xi$, namely

$$
\lambda=\frac{6}{k+2}
$$

which immediately rules out all $k>4$. For $k=4$ we have $\lambda=1$. Recall that, according to [21], this can happen if and only if $L_{4}$ is the round sphere. But we already argued that $L_{4}=S^{2} \times S^{3}$, which rules out $k=4$ also. Thus the only link that might possibly admit a Sasaki-Einstein metric with this $U(1)$ Reeb action, apart from $k=1$, 2 , is $k=3$. We shall return to the $k=3$ case in the next subsection.

\footnotetext{
${ }^{7}$ For $k=1, z_{i}, i=1,2,3$ have the smallest charge $\lambda=1$. This is consistent with the fact that $k=1$ corresponds to the link $L_{1}=S^{5}$ with its round metric.
} 
Given the contradiction, one might think that perhaps the canonical $\mathbb{C}^{*}$ action is not the critical one, in the sense of [9]. Writing

$$
F=z_{1}^{2}+u v+z_{4}^{k}
$$

there is clearly a $\left(\mathbb{C}^{*}\right)^{2}$ action generated by weights $(k, k, k, 2)$ and $(0,1,-1,0)$ on $\left(z_{1}, u, v, z_{4}\right)$, respectively. The second $U(1) \subset \mathbb{C}^{*}$ is the maximal torus of $S O(3)$ acting on the $z_{i}, i=1,2,3$, in the vector representation. It is then straightforward to compute the volume of the link as a function of the Reeb vector field

$$
\xi=\sum_{a=1}^{2} b_{a} \zeta_{a}
$$

using the character formula in [41] and taking the limit as in (2.8). We obtain

$$
V\left(b_{1}, b_{2}\right)=\frac{2 k b_{1}}{2 b_{1} \cdot k b_{1}\left(k b_{1}+b_{2}\right)\left(k b_{1}-b_{2}\right)}=\frac{1}{b_{1}\left(k b_{1}+b_{2}\right)\left(k b_{1}-b_{2}\right)} .
$$

The first component $b_{1}$ is fixed by the charge of $\Omega$, as above, to be

$$
b_{1}=\frac{3}{k+2} \text {. }
$$

According to [9], the critical Reeb vector field for the putative Sasaki-Einstein metric is obtained by setting to zero the derivative of $V\left(b_{1}, b_{2}\right)$, with respect to $b_{2}$. This immediately gives $b_{2}=0$. Thus the original weighted $\mathbb{C}^{*}$ action is indeed a critical point of the Sasakian-Einstein-Hilbert action on the link, in this 2-dimensional space of Reeb vector fields. We could have anticipated this result without computing anything. According to 9], the critical Reeb vector field could not have mixed with any vector field in the Lie algebra of a $U(1)$ subgroup of $S O(3)$, since the latter group is semisimple.

\subsection{Cohomogeneity one metrics}

It is interesting to observe that any conical Ricci-flat Kähler metric on $X_{k}$ would necessarily have $U(1) \times S O(3)$ isometry (the global form of the effectively acting isometry group will depend on $k \bmod 2$ ). This statement follows from Matsushima's theorem 7]. Specifically, Matsushima's theorem says that the isometry group of a KählerEinstein manifold ${ }^{8}\left(V, g_{V}\right)$ is a maximal compact subgroup of the group of complex

\footnotetext{
${ }^{8}$ The generalisation to orbifolds is straightforward.
} 
automorphisms of $V$. Quotienting $L_{k}$ by the $U(1)$ action, one would thus have KählerEinstein orbifold metrics on $V_{k}$ with an $S O(3)$ isometry, whose generic orbit is threedimensional. In other words, these metrics, when they exist, can be constructed using standard cohomogeneity one techniques. In fact this type of construction is very well motivated since demanding a local $S U(2) \times U(1)^{2}$ isometry is one way in which the Sasaki-Einstein metrics of [43] can be constructed (in fact they were actually found much more indirectly via M-theory [44]).

However, apart from the $k=3$ case, and of course the $k=1$ and $k=2$ cases, we have already shown that any such construction must fail. For $k=3$, the relevant ODEs that need to be solved have actually been written down in [45]. In appendix A we record these equations, as well as the boundary conditions that need to be imposed. We have been unable to integrate these equations, so the question of existence of a Sasaki-Einstein metric on $L_{3}$ remains open.

\subsection{Field theory}

In [18] a family of supersymmetric quiver gauge theories were studied whose classical vacuum moduli space reproduces the affine varieties $X_{2 p}$. These theories were argued to flow for large $N$ in the IR to a superconformal fixed point, AdS/CFT dual to a Sasaki-Einstein metric on the link $L_{2 p}$ for all $p$. Indeed, the $\mathrm{R}$-charges of fields may be computed using $a$-maximisation [14, and agree with the naive geometric computations, assuming that the Sasaki-Einstein metrics on $L_{2 p}$ exist. However, as we have already seen, these metrics cannot exist for any $p>1$. We argued in section 2 that this bound, coming from Lichnerowicz's theorem, is equivalent to the unitarity bound in the CFT. We indeed show that a gauge invariant chiral primary operator, dual to the holomorphic function $z_{4}$ that provides the geometric obstruction, violates the unitarity bound for $p>1$.

Before we recall the field theories for $k=2 p$ even, let us make a remark on the $X_{k}$ singularities when $k$ is odd. In the latter case, it is not difficult to prove that $X_{k}$ admits no crepant resolution ${ }^{9}$. That is, there is no blow-up of $X_{k}$ to a smooth manifold $\tilde{X}$ with trivial canonical bundle. In such cases the field theories might be quite exotic, and in particular not take the form of quiver gauge theories. In contrast, the $X_{2 p}$ singularities are resolved by blowing up a single exceptional $\mathbb{C P}^{1}$ [47], which leads to a very simple

\footnotetext{
${ }^{9}$ Since the link $L_{k}=S^{5}$ for all odd $k$, it follows that $\operatorname{Pic}\left(X_{k} \backslash\{r=0\}\right)$ is trivial, and hence $X_{k}$ is factorial. The isolated singularity at $r=0$ is terminal for all $k$. These two facts, together with Corollary 4.11 of [46, imply that $X_{k}$ has no crepant resolution.
} 
class of gauge theories.

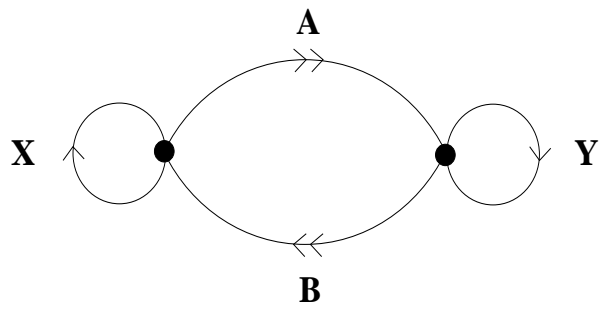

Figure 1: Quiver diagram of the $A_{1}$ orbifold gauge theory.

Consider the quiver diagram for the $\mathcal{N}=2 A_{1}$ orbifold, depicted in Figure 11. The two nodes represent two $U(N)$ gauge groups. There are 6 matter fields: an adjoint for each gauge group, that we denote by $X$ and $Y$, and two sets of bifundamental fields $A_{I}$ and $B_{I}$, where $I=1,2$ are $S U(2)$ flavour indices. Here the $A_{I}$ are in the $(N, \bar{N})$ representation of $U(N) \times U(N)$, and the $B_{I}$ are in the $(\bar{N}, N)$ representation. This is the quiver for $N$ D3-branes at the $\mathbb{C} \times\left(\mathbb{C}^{2} / \mathbb{Z}_{2}\right)$ singularity, where $\mathbb{C}^{2} / \mathbb{Z}_{2}$ is the $A_{1}$ surface singularity. However, for our field theories indexed by $p$, the superpotential is given by

$$
W=\operatorname{Tr}\left[X^{p+1}+(-1)^{p} Y^{p+1}+X\left(A_{1} B_{1}+A_{2} B_{2}\right)+Y\left(B_{1} A_{1}+B_{2} A_{2}\right)\right] .
$$

It is straightforward to verify that the classical vacuum moduli space of this gauge theory gives rise to the $X_{2 p}$ singularities. In fact these gauge theories were also studied in detail in [48, and we refer the reader to this reference for further details. The $S U(2)$ flavour symmetry corresponds to the $S O(3)$ automorphism of $X_{2 p}$.

It is a simple matter to perform $a$-maximisation for this theory, taken at face value. Recall this requires one to assign trial $\mathrm{R}$-charges to each field, and impose the constraints that $W$ has $\mathrm{R}$-charge 2 , and that the $\beta$-functions of each gauge group vanish. One then locally maximises the $a$-function

$$
a=\frac{3 N^{2}}{32}\left(2+\sum_{i} 3\left(R\left(X_{i}\right)-1\right)^{3}-\left(R\left(X_{i}\right)-1\right)\right)
$$

subject to these constraints, where the sum is taken over all $\mathrm{R}$-charges of fields $X_{i}$. One finds the results, as in 18

$$
\begin{aligned}
R(X)=R(Y) & =\frac{2}{p+1} \\
R\left(A_{I}\right)=R\left(B_{I}\right) & =\frac{p}{p+1}
\end{aligned}
$$


and central charge

$$
a\left(L_{2 p}\right)=\frac{27 p^{2} N^{2}}{8(p+1)^{3}} .
$$

This corresponds, under the AdS/CFT relation (2.32), to a Sasaki-Einstein volume

$$
\operatorname{vol}\left(L_{2 p}\right)=\frac{2 \pi^{3}(p+1)^{3}}{27 p^{2}},
$$

which agrees with the general formula (3.13). Thus an initial reaction [18] is that one has found agreement between geometric and field theory results. However, the results of this paper imply that the Sasaki-Einstein metrics on $L_{2 p}$ do not exist for $p>1$. In fact, it is clear that, upon closer inspection, the gauge invariant chiral primary operator $\operatorname{Tr} X$ (or $\operatorname{Tr} Y$ ) has $\mathrm{R}$-charge $2 /(p+1)$, which violates the unitarity bound for $p>1$. In fact, when one computes the vacuum moduli space for a single D3-brane $N=1, \operatorname{Tr} X$ is identified with the holomorphic function $z_{4}$, and the unitarity bound and Lichnerowicz bound are identical, as we argued to be generally true in section 2 .

The superpotential (4.7) can be regarded as a deformation of the $A_{1}$ orbifold theory. For $p>2$, using $a$-maximisation (and assuming an $a$-theorem), this was argued in [48. to be an irrelevant deformation (rather than a "dangerously irrelevant" operator). This is therefore consistent with our geometric results. The case $p=2$ is interesting since it appears to be marginal. If it is exactly marginal, we expect a one parameter family of solutions with fluxes that interpolates between the $A_{1}$ orbifold with link $S^{5} / \mathbb{Z}_{2}$ and the $X_{4}$ singularity with flux.

\section{$5 \quad$ Other examples}

In this section we present some further obstructed examples. In particular we examine ADE 4-fold singularities, studied in [20. All of these, with the exception of $D_{4}$ and the obvious cases of $A_{0}$ and $A_{1}$, do not admit Ricci-flat Kähler cone metrics with the canonical weighted $\mathbb{C}^{*}$ action. We also examine weighted $\mathbb{C}^{*}$ actions on $\mathbb{C}^{n}$. 


\subsection{ADE 4-fold singularities}

Consider the polynomials

$$
\begin{aligned}
H=z_{1}^{k}+z_{2}^{2}+z_{3}^{2} & A_{k-1} \\
H=z_{1}^{k}+z_{1} z_{2}^{2}+z_{3}^{2} & D_{k+1} \\
H=z_{1}^{3}+z_{2}^{4}+z_{3}^{2} & E_{6} \\
H=z_{1}^{3}+z_{1} z_{2}^{3}+z_{3}^{2} & E_{7} \\
H=z_{1}^{3}+z_{2}^{5}+z_{3}^{2} & E_{8} .
\end{aligned}
$$

The hypersurfaces $\{H=0\} \subset \mathbb{C}^{3}$ are known as the ADE surface singularities. Their links $L_{A D E}$ are precisely $S^{3} / \Gamma$ where $\Gamma \subset S U(2)$ are the finite ADE subgroups of $S U(2)$ acting on $\mathbb{C}^{2}$ in the vector representation. Thus these Gorenstein singularities are both hypersurface singularities and quotient singularities. Clearly, the links admit SasakiEinstein metrics - they are just the quotient of the round metric on $S^{3}$ by the group $\Gamma$.

The 3-fold singularities of the previous section are obtained from the polynomial $H$ for $A_{k-1}$ by simply adding an additional term $z_{4}^{2}$ (and relabelling). More generally, we may define the ADE $n$-fold singularities as the zero loci $X=\{F=0\}$ of

$$
F=H+\sum_{i=4}^{n+1} z_{i}^{2} .
$$

Let us consider the particular case $n=4$. The $\mathbb{C}^{*}$ actions, for the above cases, are generated by the weight vectors

$$
\begin{array}{rll}
\mathbf{w}=(2, k, k, k, k) & d=2 k & A_{k-1} \\
\mathbf{w}=(2, k-1, k, k, k) & d=2 k & D_{k+1} \\
\mathbf{w}=(4,3,6,6,6) & d=12 & E_{6} \\
\mathbf{w}=(6,4,9,9,9) & d=18 & E_{7} \\
\mathbf{w}=(10,6,15,15,15) & d=30 & E_{8} .
\end{array}
$$

It is then straightforward to verify that for all the $A_{k-1}$ singularities with $k>3$ the holomorphic function $z_{1}$ on $X$ violates the Lichnerowicz bound (3.23). The case $k=3$ saturates the bound, but since the link is not ${ }^{10}$ diffeomorphic to $S^{7}$ Obata's result [21] again rules this out. For all the exceptional singularities the holomorphic function $z_{2}$ on

\footnotetext{
${ }^{10}$ One can easily show that $H_{3}(L ; \mathbb{Z})=\mathbb{Z}_{k}$ for the links of the $A_{k-1}$ 4-fold singularities.
} 
$X$ violates (3.23). The $D_{k+1}$ singularities are a little more involved. The holomorphic function $z_{1}$ rules out all $k>3$. On the other hand the function $z_{2}$ rules out $k=2$, but the Lichnerowicz bound is unable to rule out $k=3$.

To summarise, the only ADE 4-fold singularity that might possibly admit a Ricciflat Kähler cone metric with the canonical $\mathbb{C}^{*}$ action above, apart from the obvious cases of $A_{0}$ and $A_{1}$, is $D_{4}$. Existence of a Sasaki-Einstein metric on the link of this singularity is therefore left open. It would be interesting to investigate whether or not there exist Ricci-flat Kähler metrics that are cones with a different Reeb action. In light of our results on the non-existence of the above Sasaki-Einstein metrics, it would also be interesting to revisit the field theory analysis of [20].

\subsection{Weighted actions on $\mathbb{C}^{n}$}

Consider $X=\mathbb{C}^{n}$, with a weighted $\mathbb{C}^{*}$ action with weights $\mathbf{v} \in\left(\mathbb{Z}_{+}\right)^{n}$. The orbit space of non-zero vectors is the weighted projective space $\mathbb{W} \mathbb{C P}_{\left[v_{1}, \ldots, v_{n}\right]}^{n-1}$. Existence of a Ricciflat Kähler cone metric on $\mathbb{C}^{n}$, with the conical symmetry generated by this $\mathbb{C}^{*}$ action, is equivalent to existence of a Kähler-Einstein orbifold metric on the weighted projective space. In fact, it is well known that no such metric exists: the Futaki invariant of the weighted projective space is non-zero. In fact, one can see this also from the Sasakian perspective through the results of [13, 9]. The diagonal action with weights $\mathbf{v}=(1,1, \ldots, 1)$ is clearly a critical point of the Sasakian-Einstein-Hilbert action, and this critical point was shown to be unique in the space of toric Sasakian metrics. Nonetheless, in this subsection we show that Lichnerowicz's bound and Bishop's bound both obstruct existence of these metrics.

The holomorphic $(n, 0)$-form on $X=\mathbb{C}^{n}$ has charge $|\mathbf{v}|$ under the weighted $\mathbb{C}^{*}$ action, which implies that the correctly normalised Reeb vector field is

$$
\xi=\frac{n}{|\mathbf{v}|} \zeta
$$

with notation as before, so that $\zeta$ is the vector field that generates $U(1) \subset \mathbb{C}^{*}$. The Lichnerowicz bound is therefore

$$
\frac{n v_{m}}{|\mathbf{v}|} \geq 1
$$

where $v_{m}$ is the (or a particular) smallest weight. However, clearly $|\mathbf{v}| \geq n v_{m}$, with equality if and only if $\mathbf{v}$ is proportional to $(1,1, \ldots, 1)$. Thus in fact

$$
\frac{n v_{m}}{|\mathbf{v}|} \leq 1
$$


with equality only in the diagonal case, which is just $\mathbb{C}^{n}$ with the canonical Reeb vector field. Thus our Lichnerowicz bound obstructs Kähler-Einstein orbifold metrics on all weighted projective spaces, apart from $\mathbb{C P}^{n-1}$ of course.

For the Bishop bound, notice that a Kähler-Einstein orbifold metric on $\mathbb{W}_{\mathbb{C}} \mathbb{P}_{\left[v_{1}, \ldots, v_{n}\right]}^{n-1}$ would give rise to a Sasaki-Einstein metric on $S^{2 n-1}$ with a weighted Reeb action. The volume of this metric, relative to the round sphere, would be

$$
V=\frac{|\mathbf{v}|^{n}}{n^{n} v}
$$

where $v=\prod_{i=1}^{n} v_{i}$ denotes the product of the weights. This may either be derived using the methods described earlier, or using the toric methods of [13]. Amusingly, (5.7) is precisely the arithmetic mean of the weights $\mathbf{v}$ divided by their geometric mean, all to the $n$th power. Thus the usual arithmetic mean-geometric mean inequality gives $V \geq 1$ with equality if and only if $\mathbf{v}$ is proportional to $(1,1, \ldots, 1)$. This is precisely opposite to Bishop's bound, thus again ruling out all weighted projective spaces, apart from $\mathbb{C P}^{n-1}$.

Thus Kähler-Einstein orbifold metrics on weighted projective spaces are obstructed by the Futaki invariant, the Bishop obstruction, and the Lichnerowicz obstruction. In some sense, these Fano orbifolds couldn't have more wrong with them.

\section{Conclusions}

The problem of existence of conical Ricci-flat Kähler metrics on a Gorenstein $n$-fold singularity $X$ is a subtle one; a set of necessary and sufficient algebraic conditions is unknown. This is to be contrasted with the case of compact Calabi-Yau manifolds, where Yau's theorem guarantees the existence of a unique Ricci-flat Kähler metric in a given Kähler class.

In this paper we have presented two simple necessary conditions for existence of a Ricci-flat Kähler cone metric on a given isolated Gorenstein singularity $X$ with specified Reeb vector field. The latter is in many ways similar to specifying a "Kähler class", or polarisation. These necessary conditions are based on the classical results of Bishop and Lichnerowicz, that bound the volume and the smallest eigenvalue of the Laplacian on Einstien manifolds, respectively. The key point that allows us to use these as obstructions is that, in both cases, fixing a putative Reeb vector field $\xi$ for the Sasaki-Einstein metric is sufficient to determine both the volume and the "holomorphic" eigenvalues using only the holomorphic data of $X$. Note that any such 
vector field $\xi$ must also be a critical point of the Sasakian-Einstein-Hilbert action of [13. 9], which in Kähler-Einstein terms means that the transverse Futaki invariant is

zero. We emphasize, however, that the possible obstructions presented here may be analysed independently of this, the weighted projective spaces at the end of section 5 being examples that are obstructed by more than one obstruction.

To demonstrate the utility of these criteria, we have provided many explicit examples of Gorenstein singularities that do not admit Sasaki-Einstein metrics on their links, for a particular choice of Reeb vector field. The examples include various quasihomogeneous hypersurface singularities, previously studied in the physics literature, that have been erroneously assumed to admit such Ricci-flat Kähler cone metrics.

We expect that in the particular case that the singularity is toric, neither Lichnerowicz nor Bishop's bound will obstruct for the critical Reeb vector field $\mathbf{b}_{*}$ of [13]. This is certainly true for all cases that have been analysed in the literature. In this case both bounds reduce to simple geometrical statements on the polyhedral cone $\mathcal{C}^{*}$ and its associated semi-group $\mathcal{S}_{\mathcal{C}}=\mathcal{C}^{*} \cap \mathbb{Z}^{n}$. For instance, given the critical Reeb vector field $\mathbf{b}_{*}$, the Lichnerowicz bound implies that $\left(\mathbf{b}_{*}, \mathbf{m}\right) \geq 1$ for all $\mathbf{m} \in \mathcal{S}_{\mathcal{C}}$. It would be interesting to try to prove that this automatically follows from the extremal problem in [13], for any toric Gorenstein singularity.

We have also explained the relevance of these bounds to the AdS/CFT correspondence. We have shown that the Lichnerowicz bound is equivalent to the unitarity bound on the scaling dimensions of BPS chiral operators of the dual field theories. In particular, we analysed a class of obstructed 3-fold singularities, parameterised by a positive integer $k$, for which, in the case that $k$ is even, the field theory dual is known and has been extensively studied in the literature. The fact that the links $L_{k}$ do not admit Sasaki-Einstein metrics for any $k>3$ supports the field theory arguments of 48. It would be interesting to know whether a Sasaki-Einstein metric exists on $L_{3}$; if it does exist, it might be dual to an exotic type of field theory since the corresponding Calabi-Yau cone does not admit a crepant resolution. For the 4-folds studied in [20], it will be interesting to analyse the implications of our results for the field theories.

\section{Acknowledgments}

We would like to thank O. Mac Conamhna and especially D. Waldram for collaboration in the early stages of this work. We would also like to thank G. Dall'Agata, M. Haskins, N. Hitchin, K. Intriligator, P. Li, R. Thomas, C. Vafa, N. Warner, and S. S.-T. Yau 
for discussions. We particularly thank R. Thomas for comments on a draft version of this paper. J. F. S. is supported by NSF grants DMS-0244464, DMS-0074329 and DMS-9803347. S.-T. Y. is supported in part by NSF grants DMS-0306600 and DMS0074329 .

\section{A Cohomogeneity one metrics}

Here we discuss the equations that need to be solved to obtain a Kähler-Einstein orbifold metric on the Fano orbifold $V_{k}$ of section 4, which recall is a hypersurface $F=z_{1}^{2}+z_{2}^{2}+z_{3}^{2}+z_{4}^{k}=0$ in the weighted projective space $\mathbb{W} \mathbb{C P}_{[k, k, k, 2]}^{3}$. The group $S O(3)$ acts on $z_{i}, i=1,2,3$, in the vector representation, and then Matsushima's theorem [7] implies that this acts isometrically on any Kähler-Einstein metric. The generic orbit is three-dimensional, and hence these metrics are cohomogeneity one. The Kähler-Einstein condition then reduces to a set of ordinary differential equations in a rather standard way.

The ODEs for a local Kähler-Einstein 4-metric with cohomogeneity one $S U(2)$ action have been written down in [45]. The metric may be written as

$$
\mathrm{d} s^{2}=\mathrm{d} t^{2}+a^{2}(t) \sigma_{1}^{2}+b^{2}(t) \sigma_{2}^{2}+c^{2}(t) \sigma_{3}^{2}
$$

where $\sigma_{i}, i=1,2,3$, are (locally) left-invariant one-forms on $S U(2)$, and $t$ is a coordinate transverse to the principal orbit. The ODEs are then [45]

$$
\begin{aligned}
& \frac{\dot{a}}{a}=-\frac{1}{2 a b c}\left(b^{2}+c^{2}-a^{2}\right) \\
& \frac{\dot{b}}{b}=-\frac{1}{2 a b c}\left(a^{2}+c^{2}-b^{2}\right) \\
& \frac{\dot{c}}{c}=-\frac{1}{2 a b c}\left(a^{2}+b^{2}-c^{2}\right)+\Lambda \frac{a b}{c}
\end{aligned}
$$

where $\Lambda$ is the Einstein constant, which is $\Lambda=6$ in the normalisation relevant for Sasaki-Einstein metrics on $L_{k}$.

The key question, given these local equations, is what the boundary conditions are. For a complete metric on $V_{k}$, the parameter $t$ must take values in a finite interval, which without loss of generality we may take to be $\left[0, t_{*}\right]$ for some $t_{*}$. At the endpoints, the principal orbit collapses smoothly (in an orbifold sense) to a special orbit. It is not difficult to work out the details for $V_{k}$, given its embedding in $\mathbb{W} \mathbb{C P}_{[k, k, k, 2]}^{3}$. One must separate $k=2 p$ even and $k$ odd. 
For $k$ odd, the principal orbit is $S O(3) / \mathbb{Z}_{2}$. This collapses to the two special orbits

$$
\begin{aligned}
B_{t=0} & =\left(S O(3) / \mathbb{Z}_{2}\right) / U(1)_{1}=\mathbb{R P}^{2} \\
B_{t=t_{*}} & =\left(S O(3) / \mathbb{Z}_{2}\right) / U(1)_{3}=\mathbb{C P}^{1}
\end{aligned}
$$

where the circle subgroups $U(1)_{1}, U(1)_{3} \subset S O(3)$ are rotations about the planes transverse to the 1 -axis and the 3 -axis, respectively, thinking of $S O(3)$ acting on $\mathbb{R}^{3}$ in the usual way. Thus the two $U(1)$ subgroups are related by a conjugation.

For $k=2 p$ even, the principal orbit is instead simply $S O(3)=\mathbb{R P}^{3}$. The two special orbits are

$$
\begin{aligned}
B_{t=0} & =S O(3) / U(1)_{1}=S^{2} \\
B_{t=t_{*}} & =S O(3) / U(1)_{3}=\mathbb{C P}^{1} .
\end{aligned}
$$

Of course, these are diffeomorphic, but the notation indicates that the second orbit is embedded as a complex curve in $V_{2 p}$, whereas $S^{2}$ is embedded as a real submanifold of $V_{2 p}$.

In both bases, with $k$ odd or $k=2 p$ even, the bolts are the real section of $V_{k}$, and the subvariety $z_{4}=0$, respectively. The latter is the image of the conic in $\mathbb{C P}^{2} \subset$ $\mathbb{W} \mathbb{C P}_{[k, k, k, 2]}^{3}$ at $z_{4}=0$, and is a locus of orbifold singularities. This is the only singular set on $V_{k}$.

The boundary conditions at $t=0$ are then, in all cases,

$$
\begin{aligned}
a(t) & =\beta+\mathcal{O}(t) \\
b(t) & =\beta+\mathcal{O}(t) \\
c(t) & =\frac{2}{k} t+\mathcal{O}\left(t^{2}\right)
\end{aligned}
$$

where

$$
\beta^{2}=\frac{k+2}{6 k} .
$$

At $t=t_{*}$, one simply requires that $a$ collapses to zero $a\left(t_{*}\right)=0$, with $b\left(t_{*}\right)=c\left(t_{*}\right)$ positive and finite. The metric functions should remain strictly positive on the open interval $\left(0, t_{*}\right)$.

The system of first order ODEs (A.2) may be reduced to a single second order ODE as follows. The change of variables $\mathrm{d} r / \mathrm{d} t=1 / c$ allows one to find the integral

$$
\frac{a}{b}(r)=-\operatorname{coth}(r)
$$


where an integration constant can be reabsorbed by a shift of $r$. Defining $f(r)=a b$, one obtains

$$
\frac{\mathrm{d}}{\mathrm{d} r} \log \left(f \frac{\mathrm{d} f}{\mathrm{~d} r}\right)=2[\Lambda f+\operatorname{coth}(2 r)] .
$$

Any solution of this equation gives rise to a solution of (A.2), using the fact that

$$
c^{2}=-\frac{\mathrm{d} f}{\mathrm{~d} r} .
$$

For $k=1, k=2$, one can write down explicit solutions to these equations and boundary conditions, corresponding to the standard metrics on $\mathbb{C P}^{2}$ and $\mathbb{C P}^{1} \times \mathbb{C P}^{1}$, respectively. For $k=1$ we have

$$
a(t)=\cos \left(t+\frac{\pi}{4}\right), \quad b(t)=\sin \left(t+\frac{\pi}{4}\right), \quad c(t)=\sin (2 t)
$$

where the range of $t$ is $0 \leq t \leq \pi / 4$. Correspondingly,

$$
f(r)=-\frac{1}{2} \tanh (2 r)
$$

with $\tan (t)=\exp (2 r)$, so that $-\infty \leq r \leq 0$.

For $k=2$ we instead have

$$
a(t)=\frac{1}{\sqrt{3}} \cos (\sqrt{3} t), \quad b(t)=\frac{1}{\sqrt{3}}, \quad c(t)=\frac{1}{\sqrt{3}} \sin (\sqrt{3} t)
$$

where the range of $t$ is $0 \leq t \leq \pi /(2 \sqrt{3})$. Correspondingly,

$$
f(r)=-\frac{1}{3} \tanh (r)
$$

with $\tan (\sqrt{3} t / 2)=\exp (r)$ and $-\infty \leq r \leq 0$.

For all $k>3$, this paper implies that there do not exist any solutions. This still leaves the case $k=3$. We have neither been able to integrate the equations explicitly, nor have our preliminary numerical investigations been conclusive. We leave the issue of existence of this solution open.

\section{References}

[1] J. M. Maldacena, "The large N limit of superconformal field theories and supergravity," Adv. Theor. Math. Phys. 2, 231 (1998) [Int. J. Theor. Phys. 38, 1113 (1999)], arXiv:hep-th/9711200. 
[2] A. Kehagias, "New type IIB vacua and their F-theory interpretation," Phys. Lett. B 435, 337 (1998), arXiv:hep-th/9805131.

[3] I. R. Klebanov and E. Witten, "Superconformal field theory on threebranes at a Calabi-Yau singularity," Nucl. Phys. B 536, 199 (1998), arXiv:hep-th/9807080.

[4] B. S. Acharya, J. M. Figueroa-O'Farrill, C. M. Hull and B. Spence, "Branes at conical singularities and holography," Adv. Theor. Math. Phys. 2 (1999) 1249, arXiv:hep-th/9808014.

[5] D. R. Morrison and M. R. Plesser, "Non-spherical horizons. I," Adv. Theor. Math. Phys. 3, 1 (1999), arXiv:hep-th/9810201.

[6] C. P. Boyer, K. Galicki, "Sasakian Geometry, Hypersurface Singularities, and Einstein Metrics," Supplemento ai Rendiconti del Circolo Matematico di Palermo Serie II. Suppl 75 (2005), 57-87 arXiv:math.DG/0405256.

[7] Y. Matsushima, "Sur la structure du groupe d'homéomorphismes analytiques d'une certaine variété kaehlérienne," Nagoya Math. J. 11 (1957), 145-150.

[8] A. Futaki, "An obstruction to the existence of Einstein Kähler metrics," Invent. Math., 73 (1983), 437-443.

[9] D. Martelli, J. Sparks, S.-T. Yau, "Sasaki-Einstein Manifolds and Volume Minimisation," arXiv:hep-th/0603021.

[10] D. Martelli and J. Sparks, "Toric geometry, Sasaki-Einstein manifolds and a new infinite class of AdS/CFT duals," Commun. Math. Phys. 262, 51 (2006) arXiv:hep-th/0411238.

[11] S.-T. Yau, "Open problems in Geometry," Proc. Symp. Pure Math. 54 (1993), $1-28$.

[12] S. K. Donaldson, "Symmetric spaces, Kähler geometry, and Hamiltonian dynamics," Amer. Math. Soc. Transl. 196 (1999), 13-33.

[13] D. Martelli, J. Sparks, S.-T. Yau, "The geometric dual of $a$-maximisation for toric Sasaki-Einstein manifolds," arXiv:hep-th/0503183, to appear in Commun. Math. Phys. 
[14] K. Intriligator and B. Wecht, "The exact superconformal R-symmetry maximizes a," Nucl. Phys. B 667, 183 (2003), arXiv:hep-th/0304128.

[15] R. L. Bishop, R. J. Crittenden, "Geometry of manifolds," Academic Press, New York, 1964.

[16] A. L. Besse, "Einstein Manifolds," Springer-Verlag, 2nd edition, 1987.

[17] A. Lichnerowicz, "Géometrie des groupes de transformations," Dunod, Paris, 1958.

[18] F. Cachazo, B. Fiol, K. A. Intriligator, S. Katz and C. Vafa, "A geometric unification of dualities," Nucl. Phys. B 628, 3 (2002) arXiv:hep-th/0110028.

[19] D. Conti, "Cohomogeneity one Einstein-Sasaki 5-manifolds," arXiv:math.DG/0606323.

[20] S. Gukov, C. Vafa and E. Witten, "CFT's from Calabi-Yau four-folds," Nucl. Phys. B 584, 69 (2000) [Erratum-ibid. B 608, 477 (2001)] arXiv:hep-th/9906070.

[21] M. Obata, "Certain conditions for a Riemannian manifold to be isometric to a sphere," J. Math. Soc. Japan 14 (1962), 333-340.

[22] S. Minakshisundaram and A. Pleijel, "Some Properties of the Eigenfunctions of the Laplace-Operator on Riemannian Manifolds," Can. J. Math. 1 (1949).

[23] J. Kollár, "Rational Curves on Algebraic Varieties," Springer-Verlag. Ergebnisse der Math. vol 32, 1996.

[24] J.-M. Hwang, "On the degrees of Fano four-folds of Picard number 1," J. Reine Angew. Math. 556 (2003), 225-235.

[25] S. Gubser, N. Nekrasov and S. Shatashvili, "Generalized conifolds and four dimensional $\mathrm{N}=1$ superconformal theories," JHEP 9905, 003 (1999) arXiv:hep-th/9811230.

[26] S. S. Gubser, I. R. Klebanov and A. M. Polyakov, "Gauge theory correlators from non-critical string theory," Phys. Lett. B 428, 105 (1998) arXiv:hep-th/9802109.

[27] E. Witten, "Anti-de Sitter space and holography," Adv. Theor. Math. Phys. 2, 253 (1998) arXiv:hep-th/9802150. 
[28] I. R. Klebanov and E. Witten, "AdS/CFT correspondence and symmetry breaking," Nucl. Phys. B 556, 89 (1999) arXiv:hep-th/9905104.

[29] V. Balasubramanian, P. Kraus and A. E. Lawrence, "Bulk vs. boundary dynamics in anti-de Sitter spacetime," Phys. Rev. D 59, 046003 (1999) arXiv:hep-th/9805171.

[30] A. Ceresole, G. Dall'Agata, R. D'Auria and S. Ferrara, "Spectrum of type IIB supergravity on AdS(5) x T(11): Predictions on $\mathrm{N}=1$ SCFT's," Phys. Rev. D 61, 066001 (2000) arXiv:hep-th/9905226.

[31] D. Fabbri, P. Fre, L. Gualtieri and P. Termonia, "M-theory on AdS(4) x M(111): The complete $\operatorname{Osp}(2 \mid 4)$ x SU(3) x SU(2) spectrum from harmonic analysis," Nucl. Phys. B 560, 617 (1999) arXiv:hep-th/9903036.

[32] D. Fabbri, P. Fre', L. Gualtieri, C. Reina, A. Tomasiello, A. Zaffaroni and A. Zampa, "3D superconformal theories from Sasakian seven-manifolds: New nontrivial evidences for $\operatorname{AdS}(4) / \mathrm{CFT}(3), "$ Nucl. Phys. B 577, 547 (2000) arXiv:hep-th/9907219.

[33] H. J. Kim, L. J. Romans and P. van Nieuwenhuizen, "The Mass Spectrum Of Chiral N=2 D = 10 Supergravity On $S^{5}$," Phys. Rev. D 32, 389 (1985).

[34] L. Castellani, R. D'Auria, P. Fre, K. Pilch and P. van Nieuwenhuizen, "The Bosonic Mass Formula For Freund-Rubin Solutions Of D = 11 Supergravity On General Coset Manifolds," Class. Quant. Grav. 1, 339 (1984).

[35] R. D'Auria and P. Fre, "Universal Bose-Fermi Mass Relations In Kaluza-Klein Supergravity And Harmonic Analysis On Coset Manifolds With Killing Spinors," Annals Phys. 162, 372 (1985).

[36] M. Henningson and K. Skenderis, "The holographic Weyl anomaly," JHEP 9807 (1998) 023 arXiv:hep-th/9806087.

[37] S. S. Gubser, "Einstein manifolds and conformal field theories," Phys. Rev. D 59, 025006 (1999) arXiv:hep-th/9807164.

[38] J. P. Gauntlett, D. Martelli, J. Sparks and D. Waldram, "Supersymmetric AdS(5) solutions of type IIB supergravity," Class. Quant. Grav. 23, 4693 (2006) arXiv:hep-th/0510125. 
[39] R. C. Randell, "The homology of generalized Brieskorn manifolds", Topology 14 (1975), no. 4, 347-355.

[40] A. Bergman, C. P. Herzog, "The Volume of some Non-spherical Horizons and the AdS/CFT Correspondence," JHEP 0201 (2002) 030, arXiv: arXiv:hep-th/0108020.

[41] N. Nekrasov and S. Shadchin, "ABCD of instantons," Commun. Math. Phys. 252, 359 (2004) arXiv:hep-th/0404225.

[42] I. Smith, R. P. Thomas, "Symplectic surgeries from singularities," Turkish J. Math. 27 (2003), 231-250 arXiv:math.SG/0212213.

[43] J. P. Gauntlett, D. Martelli, J. Sparks and D. Waldram, "Sasaki-Einstein metrics on $S^{2} \times S^{3}$," Adv. Theor. Math. Phys. 8, 711 (2004) arXiv:hep-th/0403002.

[44] J. P. Gauntlett, D. Martelli, J. Sparks and D. Waldram, "Supersymmetric $\mathrm{AdS}_{5}$ solutions of M-theory," Class. Quant. Grav. 21, 4335 (2004) arXiv:hep-th/0402153.

[45] A. S. Dancer, I. A. B. Strachan, "Kähler-Einstein metrics with $S U(2)$ action," Math. Proc. Camb. Phil. Soc. 115, 513 (1994).

[46] J. Kollár, "Flops," Nagoya Math. J. 113 (1989), 15-36.

[47] H. B. Laufer, "On $\mathbb{C P}^{1}$ as an exceptional set", in Recent developments in several complex variables, Princeton University Press and University of Tokyo Press, 1981.

[48] R. Corrado and N. Halmagyi, "N = 1 field theories and fluxes in IIB string theory," Phys. Rev. D 71, 046001 (2005) arXiv:hep-th/0401141. 